\title{
Complete genome sequence of a novel partitivirus from a wild brassicaceous plant, Arabidopsis halleri
}

\section{AUTHOR(S):}

Kamitani, Mari; Okuno, Tetsuro; Kudoh, Hiroshi

\section{CITATION:}

Kamitani, Mari ... [et al]. Complete genome sequence of a novel partitivirus from a wild brassicaceous plant, Arabidopsis halleri. Archives of Virology 2020, 165: 2091-2094

\section{ISSUE DATE:}

2020

URL:

http://hdl.handle.net/2433/254202

\section{RIGHT:}

This is a post-peer-review, pre-copyedit version of an article published in 'Archives of Virology'. The final authenticated version is available online at: https://doi.org/10.1007/s00705-020-04670-y.; The full-text file will be made open to the public on 12 June 2021 in accordance with publisher's 'Terms and Conditions for Self-Archiving'.; This is not the published version. Please cite only the published version.; この論文は出版社版でありません。引用の際には出版社版 をご確認ご利用ください。 
$1 \quad$ Title

2 Complete genome sequence of a novel partitivirus from a wild brassicaceous plant, Arabidopsis

3 halleri

4

$5 \quad$ Author list

6 Mari Kamitani ${ }^{1,2}$

7 Tetsuro Okuno ${ }^{1}$

$8 \quad$ Hiroshi Kudoh ${ }^{2}$

$9 \quad{ }^{1}$ Faculty of Agriculture, Ryukoku University, Yokotani1-5, Seta Oe-cho Otsu, Shiga 520-2194,

10 Japan

$11{ }^{2}$ Center for Ecological Research, Kyoto University, Hirano 2-509-3, Otsu, Shiga 520-2113,

12 Japan

13

14 Corresponding author

15 Mari Kamitani

$16 \quad$ Email: kamitani.mari.86a@kyoto-u.jp

17 Hiroshi Kudoh

18 Email: kudoh@ecology.kyoto-u.ac.jp

19 


\section{Abstract}

27 Two contigs with high similarity to partitivirus species were identified by de novo assembly of sequences obtained by the RNA-Seq on a wild brassicaceous plant, Arabidopsis halleri subsp. gemmifera. Here, we determined the full-genome sequence of a putative novel partitivirus. Excluding the poly-A tail, it consisted of two RNA genome segments of 1912 and $1769 \mathrm{bp}$, which predicted to encode a 585-amino-acid-long putative RNA-dependent RNA polymerase (RdRp) and a 487-amino-acid-long putative capsid protein (CP), respectively. Phylogenetically, this virus belongs to the genus Alphapartitivirus and is most closely related to Raphanus sativus partitivirus 1 reported from radish. We propose the name of the novel virus, Arabidopsis halleri partitivirus 1 (AhPV1). 
51 Knowledge on viruses in wild plants is quite limited, although wild plants may act as reservoirs of crop diseases. Recently, increasing numbers of studies are trying to reveal plant virus diversity in nature using data obtained from high-throughput sequencing technologies. Partitiviruses are one of the most frequently identified viruses in such surveys $[3,7]$. They possess two essential dsRNA genome segments, RNA1 and RNA2, which encode RNAdependent RNA polymerase (RdRp) and capsid protein (CP), respectively [5]. Members of the family Partitiviridae have been reported not only from plants, but also from fungi and protozoa. Partitiviruses are known as cryptic viruses that cause few or no visible symptoms in their hosts $[5,6]$. In our previous study, we conducted virus survey in a brassicaceous plant, Arabidopsis halleri subsp. gemmifera (A. halleri), using RNA-Seq with Illumina HiSeq 2500 and de novo assembly [2]. In total, 68 plants were sampled and each of the leaf samples was barcoded with different index-sequence and sequenced using 1 lane of Illumina HiSeq 2500. Infection of Turnip mosaic virus, Cucumber mosaic virus, Brassica yellows virus were determined and in de novo assembly, we detected two novel sequences with high similarity to partitiviruses [2]. The two sequences were also detected by RT-PCR. Both sequences had open reading frames encoding a putative $\mathrm{RdRp}$ and $\mathrm{CP}$, respectively and were always detected simultaneously in the partitivirus-infected plants [2]. These sequences were detected from 56 plant-individuals in the examined 68 plants. In this study, we determined the full-length genome sequence of the putative partitivirus by conducting a rapid amplification of cDNA ends (RACE) analysis on the 5' and 3' ends of the genome segments. Total RNA was extracted from the leaves of the virus-infected $A$. halleri plants using Maxwell 16 LEV Plant RNA Kit (Promega, WI, USA). Reverse transcription (RT) was conducted with oligo-dT primers and Superscript IV Reverse Transcriptase (Thermo Fisher Scientific Inc., MA, USA). The $3^{\prime}$ and 5' ends of the two genome segments were amplified using the SMARTer RACE 5'/3' Kit (Takara-bio, Japan) according to the manufacturer's instruction. The rest middle-part 
of the two segments were also amplified using primers constructed based on the sequence determined by $3^{\prime}$ and 5' RACE (Supplementary Figure 1). The whole-genome sequence was determined by Sanger sequencing (Eurofin Genomics, Luxembourg). Both strands of the AhPV1 ds-RNA genome were detected by strand specific RT-PCR (Supplementary Figure 2). These sequences were deposited as the complete genome sequence of AhPV1 in the National Center of Biotechnology Information (NCBI) GenBank database with accession numbers MT155793 and MT155794.

The two segments of the AhPV1 genome were 1912 and 1769 bp in length excluding poly-A tail and encoded putative RdRp and CP, respectively (Fig. 1). The lengths of the deduced aminoacid (aa) sequences of RdRp and CP were 585 aa and 487 aa, respectively. The lengths of the untranslated regions at $5^{\prime}$ and $3^{\prime}$ ends of the segments, respectively, were $77 \mathrm{bp}$ and $77 \mathrm{bp}$ in RNA1, and 115 bp and 190 bp in RNA2 (Fig. 1). A phylogenetic analysis using the deduced amino-acid sequence of RdRp of this virus and reported partitiviruses [5] indicated that this virus belongs to the genus Alphapartitivirus (Figure 2) and is most closely related to Raphanus sativus partitivirus 1 (RsPV1), which has been reported from a brassicaceous crop plant. The aa sequence identity of RdRp between RsPV1 and AhPV1 was 80.6\%. The sequences of untranslated region (UTR) of RNA1 from RsPV1 and AhPV1 also had high similarity (Supplementary Figure 3). The nucleotide identity between RsPV1 and AhPV1 was 61.0\% (50 sites against aligned 82 nucleotide-sites) and 65.4\% (53 sites against aligned 81 nucleotidesites) for 5' UTR and 3' UTR, respectively. The conserved A/T-rich regions observed in the 3' UTR might contain a polyadenylation signal of these viruses. The CP aa sequence of RsPV1 has not been reported; however, the CP aa sequence of AhPV1 showed 32.7\% identity with that of Rosellinia necatrix partitivirus 2 (RnPV2). The identities of $5^{\prime}$ and $3^{\prime}$ UTRs between the viruses were $35.7 \%$ and $44.9 \%$, respectively. Segmented viruses including partitivirus have the capacity to exchange their genome segments in co-infection through reassortment, which drives 
rapid evolutionary-changes [4]. We compared phylogenetic locations of RNA1 (RdRp) and RNA2 (CP) of AhPV1 to analyse whether AhPV1 could be derived from reassortment among disparate species or not (Figure 2 and Supplementary Figure 4). In the phylogenetic tree of CP, four genera of partitivirus did not always form single clades as observed in a previous study [1]. Among partitiviruses whose CP sequences were reported, AhPV1 was most closely related to RnPV2 (Supplementary Figure 4). Therefore, phylogenetic locations of the two genome segments of AhPV1 were similar and no obvious evidence of reassortment was observed. Plantinfecting partitiviruses are known to be transmitted intracellularly during cell division and to persistently infect hosts. Horizontal transmission via vectors has not been reported, while vertical transmission through seeds has been reported widely [3, 8]. We determined the seed transmission rate of AhPV1 using 22 seedlings from the surface-sterilised seeds obtained from three AhPV1-infected wild A. halleri plants. The AhPV1 infection was detected in 16 out of 22 seedlings by RT-PCR using primers designed to amplify RdRp sequences; forward ATGAAGAACACCGTCGTTCTC, and reverse GACTTCAGTTTCCCGTCATAC. This result indicates that the seed transmission rate was $72.7 \%$.

In summary, we characterized a putative novel virus from a wild brassicaceous plant, and it was considered to belong to the genus Alphapartitivirus. The criterion for species demarcation in the genus Alphapartitivirus is that the two species have less than $90 \%$ and $80 \%$ identity between the amino-acid sequences of their RdRps and CPs, respectively [8]. Considering this criterion, we regarded AhPV1 as a novel species of the genus Alphapartitivirus. Because the family Partitiviridae includes both plant and fungal viruses, improving our knowledge about these viruses is a promising way to understand the evolutionary relationships or horizontal transmission of viruses between plants and fungi.

\section{Acknowledgement}


126 We thank G. Yumoto for his help in field sampling.

127

128 Declarations

129 Funding

130 This work was supported by JSPP KAKENHI Grant Number 18K05658 to TO, Japan Science

131 and Technology Agency (JST) CREST no. JPMJCR15O1 to HK and Grant-in-Aid for Japan

132 Society for the Promotion of Science Fellows 19J01031 to MK.

\section{Competing interests}

134 The authors declare that they have no conflict of interest.

135 Availability of data and material

136 The full-genome sequence of AhPV1 were deposited in the NCBI database with accession 137 numbers MT155793 and MT155794..

138 Authors' contribution

139 M.K. and H.K conducted field sampling. M. K. conducted the laboratory experiment and wrote 140 the manuscript. M.K., T.O. and H.K discussed the results. All authors approved the manuscript. 
(A)

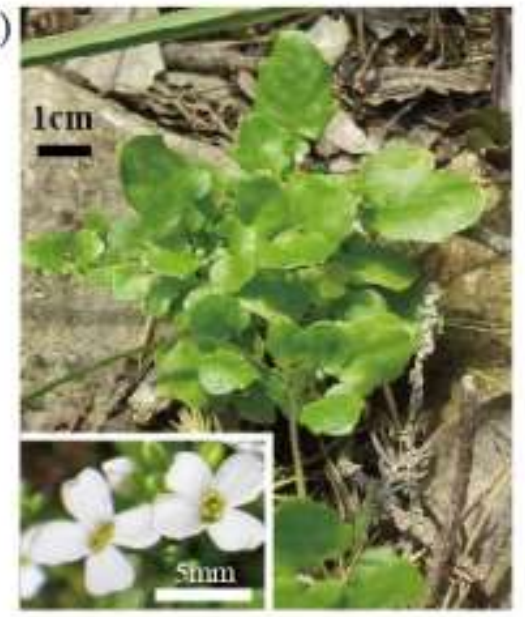

(B)

RNA1 1912 bp

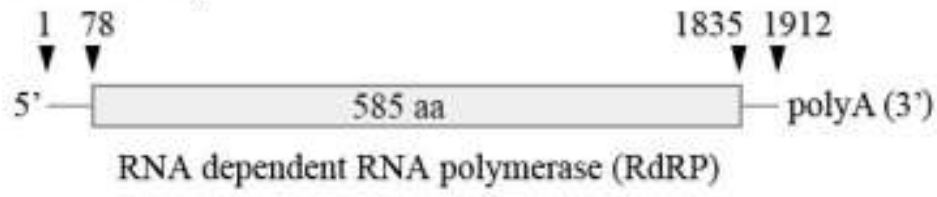

RNA2 1769 bp

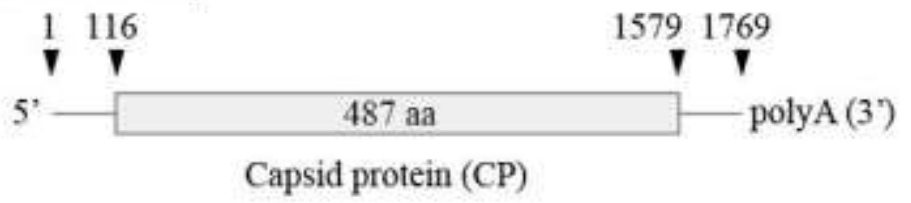

155 Figure 1 Diagram of the genome organization of Arabidopsis halleri partitivirus 1(AhPV1) detected from Arabidopsis halleri

(A) Representative individuals of Arabidopsis halleri infected by the novel partitivirus without apparent symptoms under natural environments. (B) Schematic diagram of AhPV1 genome.

159 The putative ORFs and untranslated regions were indicated by boxes and lines, respectively. 


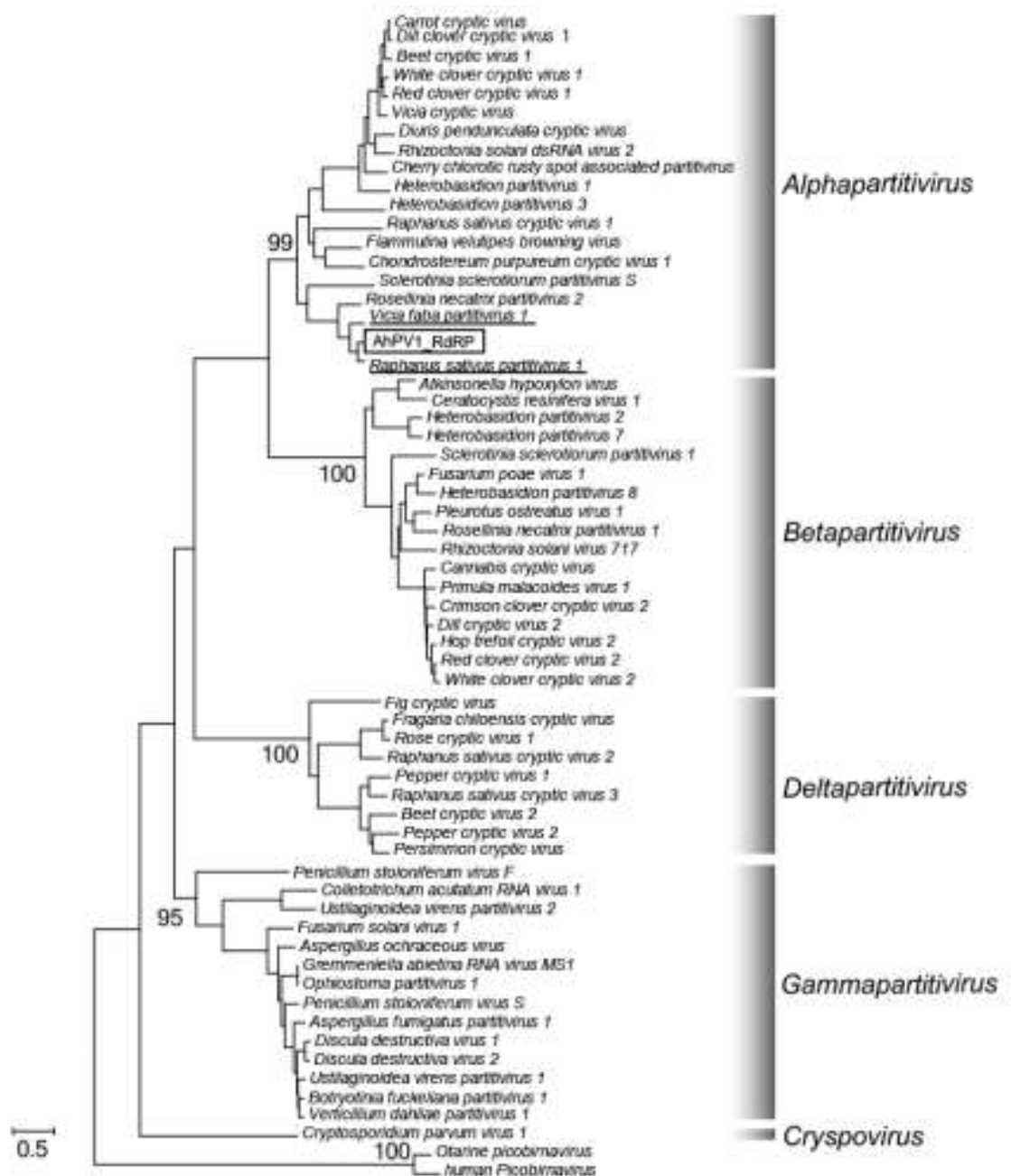

Figure 2 Phylogenetic location of Arabidopsis halleri partitivirus 1(AhPV1) based on

\section{RdRp aa sequences.}

Phylogenetic location of AhPV1 (boxed) was shown. The phylogenetic tree was constructed by MEGA7 using maximum likelihood method based on the Le_Gascuel_2008 model. Corresponding viruses and the accession numbers in NCBI database are listed in Supplementary Table 1. Vertical lines correspond to the four genera of partitivirus and Cryspovirus was added to as a fifth genus of partitivirus. Otarine picobirnavirus and human Picobirnavirus were used as outgroups. Underlining indicates related Alphapartitivirus for which the CP sequence is unknown. Numbers beside the clades represent bootstrap values for the branches supporting monophyly of genera. 


\section{References}

173 1. Rosana B, Dirk S, Edgar M (2007). Molecular characterization and detection of Vicia cryptic virus in different Vicia faba cultivars. Arch Virol 152:1477-88

2. Kamitani M, Nagano AJ, Honjo MN, Kudoh H (2016) RNA-Seq reveals virus-virus and virus-plant interactions in nature. FEMS Microbiol Ecol 92

3. Li L, Liu J, Zhang Q, Fu R, Zhu X, Li C, Chen J (2016) Seed-borne viral dsRNA elements in three cultivated Raphanus and Brassica plants suggest three cryptoviruses. Can J Microbiol 62:287-295

4. McDonald SM, Nelson MI, Turner PE, Patton JT (2016) Reassortment in segmented RNA viruses: mechanisms and outcomes. Nat Rev Microbiol 14(7): 448-460

5. Nibert ML, Ghabrial SA, Maiss E, Lesker T, Vainio EJ, Jiang D, Suzuki N (2014) Taxonomic reorganization of family Partitiviridae and other recent progress in partitivirus research. Virus Res 188:128-141

6. Roossinck MJ (2010) Lifestyles of plant viruses. Philos Trans R Soc Lond B Biol Sci 365:1899-1905

7. Roossinck MJ (2015) Plants, viruses and the environment: Ecology and mutualism.

8. Vainio EJ, Chiba S, Ghabrial SA, Maiss E, Roossinck M, Sabanadzovic S, Suzuki N, Xie J, 


\section{>AhPV1 RNA1}

TCAAAATATAGGAAGGGAACCTACAAAGCTTCCATCTTTCTCTATTTTCTCTCAACAGACATTCTGTTCACAC ACTAATGAAGAACACCGTCGTTCTCGAACCACTGCCATCGCTGGCCAGGCCCATTTATGGAGATACCGACCC Annealing site of 5'RACE primer (R) Annealing site of PCR primer (F)

AGGTCGAAATCCAGCCTACCAGAGTACAGTAGACCACGCACTCAGGCGACTCCTCACAGCTGAAGAGTTCA ACATTGTCGTCAATGGCTACCGACGTTCCCCTTGGAATGAAGACGCCCTAACCGCCGATATTGAAAAGCTCA ACTCTGACTATCACCACGTCAATAAAGATGAGCATTACTACAAAGCTATTGAACATACAAAGAAATTGTTCAC ACCAAAGGAGAAATTAAGACCCGTGCATTTTAATGATCTACGTCACTACCCATGGCAATTGTCAACGAGTATT GGCGCTCCATTCGCGACAAGCGAAAAGTGGAAGGATTATATTAATCAGAAGTATGACGGGAAACTGAAGTCT AGAGACTTTAAAGACCTATTCAAAGAAACTCATGGAGTTTCGCTTGAACCATACATGATCGATAGACGCTTAT CAAAGCGTAACTTCTACAATGAAATGTTCTACATTAATCGAATTAATATTCATCACATTAAAGATGGATGGACA ACGAATCCAGCAGGACACGATTTACGTTACTGGCATACTGCACACGCAAGACAACACTTAGTTGAAGCCGG AGACGAAGACAAAGTCCGACTAGTATTCGGTGCACCTTCTACCTTACTAATGGCCGAGCTCATGTTCATTTGG CCGATCCAGACTAGTTTACTAGCACGTGGATCTTCTTCGCCAATGTTATGGGGCTACGAAACCACTACAGGGG GATGGTCCCGGTTATACAACTGGGCATATTCTGCCCTTCCCAGATTCGGAGCCGTCGCTACCCTTGATTGGAG TAGATTCGACAAAGACGCTCGACATACTGTAATCACAGACATACATGATTTAATCATGCGCCCAATGTTCGAC TTCAATTCAGGCTACCACCCAACTATAATCAACCCAAGATCTAATCCAGACCCGCAAAGGCTGGAGAATCTAT GGAATTGGATGAAGAATGCAATCCTAACGACCCCTCTGCTGCTGCCAGATGGGACGAGACTACAATTCCAAC ATTCTGGAATTTATTCAGGATACTTTCAAACACAGATATTAGACTCAATGTATAATTGCGTCATGATATTTACCG TTCTTTCAAGAATGGGATTCGATTTAAACTCAGTTGCTATTAAAGTACAAGGAGATGACTCACTCATCTTACT CAGCCACAGCTACACTTTCTTGCAACATTCGTTTCTGACTACGTTTGCACACCACGCTGCCGTATATTTCGGC TCGACGCTGAACGTAAAGAAAAGCGAGCTTTTACCATCACTAGAAGACGCTGAAGTTTTGAGATACAGAAA TCATGGTATGATGCCATATCGTGAAGAACTTCAACTACTAGCAATGCTACGACACCCAGAAAGGACTGCCTC ACTCTCAGCCCTCATGGCACGAAGCATCGGAATAGCATACGCTAACTGCGGAAACTACACCCGTGTACACCA CATCTGCGAGGATATCCACAATTACCTAAAAGGGATTGGGGTTAAGCCAGACGCATTTGGATTACCAGGTGG ATTAAGGTTTCGAAAGAACTACCTCCCCTCTTATGAAGAAATCGACATCAGCCACTTCCCAACATGGCTAGA GACCGTCGAACGCTTACTAGACCCCTCAAGACCTCTGCTTACCAACAAGCACTGGCCTACCACTCACTTT Annealing site of PCR primer (R) Annealing site of 3'RACE primer (F)

TTCGGTATCCCCGGAGAGTCCTAGGATAGGACGTTATTAATATTTATTTTTACGTTTCTGCCCTTTGTAACTAA AAATTTAAAATAATAATAATAATATATAAAAAAAAAAAAAAAA

\section{$>$ AhPV1 RNA2}

TAAAATAACTGGAGAAATTACTACCAATTTCAAATTCCCACGTTATAATTAACCCAACAGATATTCTGTTCCC TTATATAGCTCCCCACTCACCACAACAACTTGTCTACTTACAATGTCGATGAAAAGAAAGTCAAGGCCTAGT TCTTCAACAAAAGATTTAGAGGTAGAAGACACCTATCTCAAACAATCTGGACTTGATTCAATGAATAAGCTC GAACCAGTCGAGCAATCAAAAGACGAAGAGACTACCAAAGTCTCCATGCTCCCCACCGCTTCTACTGCTATA Annealing site of RACE primer (R) Annealing site of PCR primer (F) ATCGCCCCGCGTAAACTAACTGCGGAAGATTTTAGCTCTAAACGTAAGCCGGATCAAACGTCCGCTGTTAGC CCATTCTTTGGGTTCCTTAGGACCCACATCCTACACCCTACACAGGGCAGGCTCTCACACTACTACCCTTCCT GCCACATGATGGACTACATTCTTCACTCTATCAATTCAACTCTCTGTGATAATTACTACTTCAAGAGAGAAACT CCAAACTACCACCCTTACATTCTCCGACTCTACTTCGGAGTTCTTTTCTGGGTTCAGTGCTTGCGCGCTGGAA ATGATGTTCAGGTCATTAATGACCTACACTACGATTTCTTGCAGCGTTTCCTAGACTGCAATCCTCTCGAGTCT TTGGCCATTCCAGGCCCACTTCTCGGACTCTTCAAGACACTCTGCTCCTCCCAGCCAGAGTTCCCACATTATG GGAAAGTTTACCCCCGCATTCCTGCGAGCCCAGGACCAAGACGCCGAGACATGTTCTCAAAGAACGTCCCA AGCGCCCAATTCTTACCCAACGTTCCTGGTATTTTCGCACTCATCCACCATCTCCACGGACTTTCTGAGGGTG AACACCCGATTTACCCGAAAAGAAAAAGACACATTCCTGTCACTGAGGAGGCCAGTAACTTCGGTTTCAAG GCCTTCGCCGCTTTTCCAAACAGAATCCAGCGCGATCGTTGGATGGTTAGCTCCCCCGGCCTCCAGTATCCCT GTGAAGCCGACATGAAGATGAATGAAGCGTTCGCTGAACGTTTCTATGATTTTGACTTTCCTGCCTTTAACGC AGATGACAATCTCTCCACCATCACCAACTTCCTCCACATGAGGAAAAGTATGGCTTGGTTCATCCGGGCCAA GGAAGTCGCCTGCTCGGCCGCTAGATTCTTTTCAGACTCTGGCACTCTCGCCGACTGTTCTCCACACGGTCT GGTCTCAAACCAGATCATTGTTGCGATTACTCCTCCACCTGAGGAGACTTTTGCTGATCCCCGCTTCTCCGCC GATCCAAGAGCCCTCTATCCCTTCAGTTTCAAGCTGAAGAGCACCGCCCACAACCTCCCCCCACTTGCGGAA GCTGCCGCAGCCTTCTCCCAGACGCACATCCGGATTTTTCCGGAATACCCGTTGGCCGGAAACTTTGGTCAA AAGACCGACGAATCAGGCCCCTTTTGGGACATCAGGCCCATTGGCTCCAGCCCCACCGACGACACCTCCTA CCTCACCATCCCACCCATGGTCAAGGCAGCACTCATCGAGAAAGGCTCCAGCCGTTAGGAGTCATGCACTG Annealing site of PCR primer (R) Annealing site of 3’RACE primer $(F)$ ACAGACATCAGCCGCTGACCACCTTTTTTCTTTTTCCTAGTATCTCGGATTTAGAAAGCAGCAATTTTACAAA AGCAGTCCCATTCGGACTAGCTGCTTTTTCTTACGCATTTGCGTTTGCTTTTCCCTTTTAGTATTCCTTCTTTTA TTACTAAATGTATAACTTAAATTAAAAAGAAAAAAAAAAAAAAAA

\section{Supplementary Figure 1 Complete nucleotide sequence of the two genome-segments of AhPV1 and location of primers used to amplify the whole sequences.}

Start codons and stop codons are marked in boxes. Primers used for 5'RACE or 3'RACE is indicated by double underlines. Shaded sequences represent PCR primers to amplify the rest middle-part of the segments. Bold "A" characters at 3'end of the segments represent sequences that are regarded as poly-A tails. 
(A)

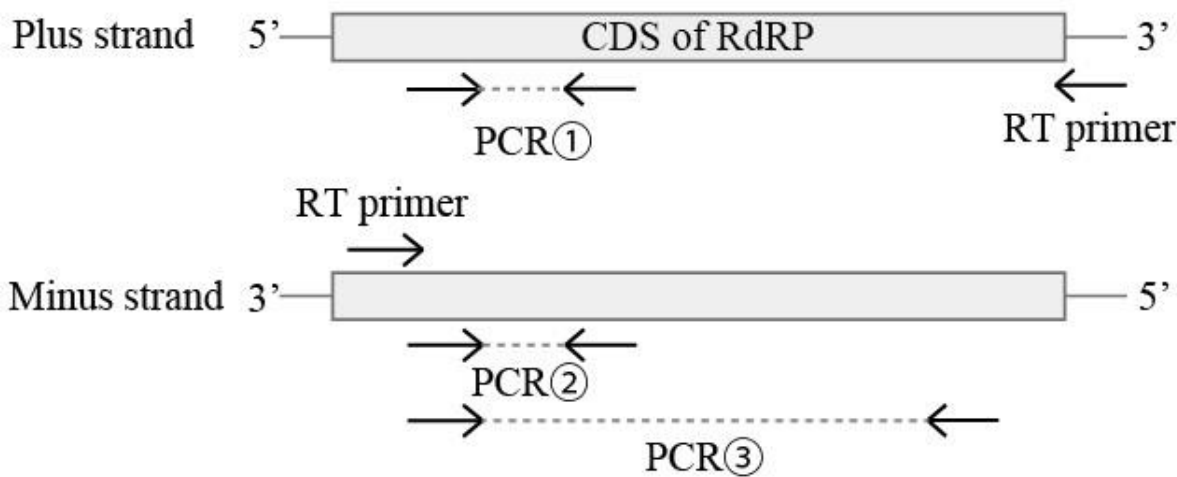

(B)

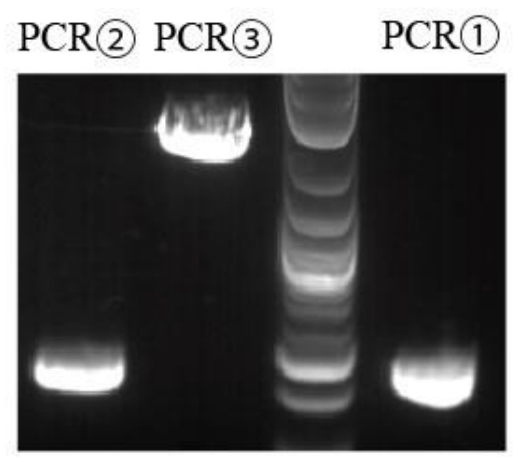

Supplementary Figure 2 Detection of both strands of AhPV1 dsRNA

(A) Schematic diagrams of ds RNA of AhPV1 RNA1 was shown. Arrows represents RT and PCR primers used for strand-specific RT-PCR. (B) Amplified fragments on 1\% agarose gel were shown. 
(A) RNA1 5, UTR

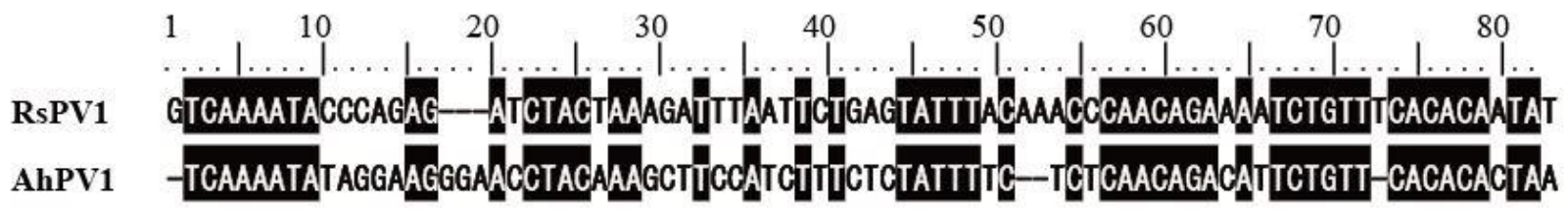

(B) RNA1 3' UTR

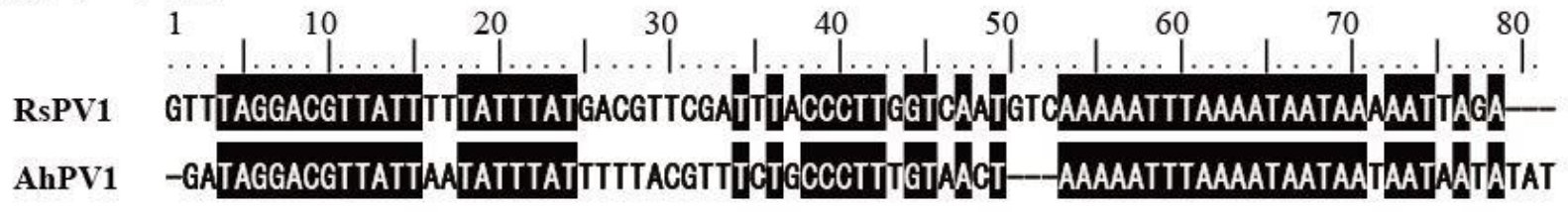

Supplementary Figure 3 Comparison of untranslated region of RNA1 fromRsPV1 and AhPV1. Comparison of 5' UTR (A) and 3' UTR (B) were shown. Common nucleotide-residue at each site is shaded by black. The sequence of RNA2 was not reported for RsPV1. 


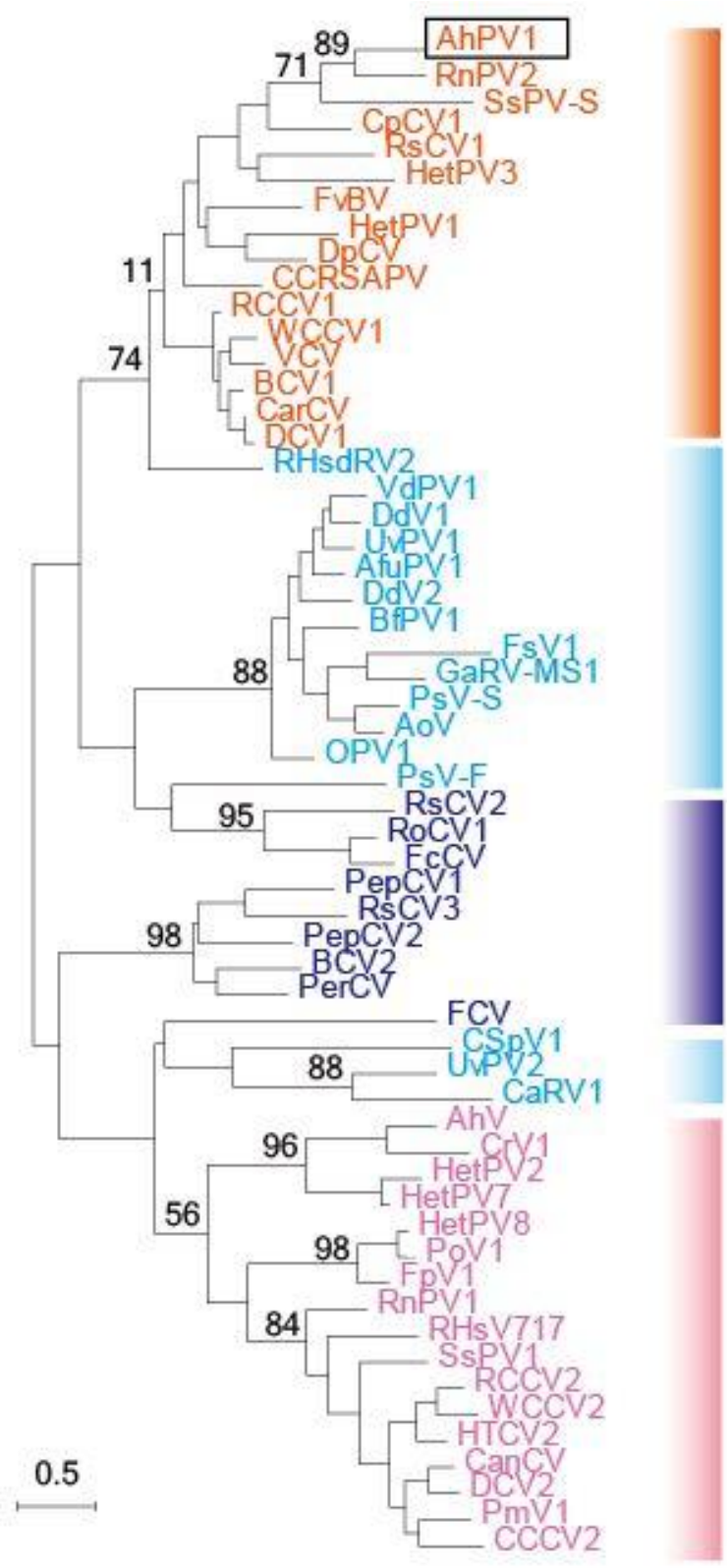

Alphapartitivirus

Gammapartitivirus

Deltapartitivirus

Gammapartitivirus

Betapartitivirus

Supplementary Figure 4 Phylegenetic analysis of AhPV1 based on CP aa sequences.

Phylogenetic location of AhPV1 (boxed) was shown. The tree was constructed by MEGA7 using maximum likelihood method based on the Jones-Taylor-Thrnton model. Viruses were indicated by their abbreviation listed in Supplementary Table 1. Vertical lines and colours correspond to the genera. Numbers beside the clades represent bootstrap values for the branches. 
Supplementary Table 1 List of the species* used in phylogenetic analysis

\begin{tabular}{|c|c|c|c|c|}
\hline No. & $\begin{array}{l}\text { Reference } \\
\text { strain } \\
\text { abbreviation }\end{array}$ & Species name & $\begin{array}{l}\text { GenBank accession no. } \\
\text { dsRNA1(RdRP) }\end{array}$ & $\begin{array}{l}\text { GenBank accession no. } \\
\text { dsRNA2(CP) }\end{array}$ \\
\hline 1 & AhPV1 & Arabidopsis halleri partitivirus 1 & LC151461 & LC151462 \\
\hline 2 & RsPV1 & Raphanus sativus partitivirus 1 & KT285019 & - \\
\hline 3 & VfPV1 & Vicia faba partitivirus 1 & DQ910762 & - \\
\hline 4 & AfuPV1 & Aspergillus fumigatus partitivirus 1 & FN376847.3 & FN398100.2 \\
\hline 5 & AhV & Atkinsonella hypoxylon virus & L39125 & L39126 \\
\hline 6 & AoV & Aspergillus ochraceous virus & EU118277 & EU118278 \\
\hline 7 & BCV1 & Beet cryptic virus 1 & EU489061 & EU489062 \\
\hline 8 & $\mathrm{BCV} 2$ & Beet cryptic virus 2 & HM560703 & HM560702 \\
\hline 9 & BfPV1 & Botryotinia fuckeliana partitivirus 1 & AM491609 & AM491610 \\
\hline 10 & CanCV & Cannabis cryptic virus & JN196536 & JN196537 \\
\hline 11 & $\mathrm{CarCV}$ & Carrot cryptic virus & FJ550604 & FJ550605 \\
\hline 12 & CaRV1 & Colletotrichum acutatum RNA virus 1 & KC572132 & KC572133 \\
\hline 13 & CCCV2 & Crimson clover cryptic virus 2 & JX971982 & JX971983 \\
\hline 14 & CCRSAPV & Cherry chlorotic rusty spot associated partitivirus & AJ781401 & AJ781402 \\
\hline 15 & CpCV1 & Chondrostereum purpureum cryptic virus 1 & AM999771 & AM999772 \\
\hline 16 & CrV1 & Ceratocystis resinifera virus 1 & AY603052 & AY603051 \\
\hline 17 & $\mathrm{CSpV} 1$ & Cryptosporidium parvum virus 1 & U95995 & U95996 \\
\hline 18 & DCV1 & Dill clover cryptic virus 1 & KF484726 & KF484727 \\
\hline 19 & DCV2 & Dill cryptic virus 2 & JX971984 & JX971985 \\
\hline 20 & DdV1 & Discula destructiva virus 1 & AF316992 & AF316993 \\
\hline 21 & $\mathrm{DdV} 2$ & Discula destructiva virus 2 & AY033436 & AY033437 \\
\hline 22 & $\mathrm{DpCV}$ & Diuris pendunculata cryptic virus & JX156424 & JX891460 \\
\hline 23 & $\mathrm{FcCV}$ & Fragaria chiloensis cryptic virus & DQ093961.2 & DQ355440 \\
\hline 24 & $\mathrm{FCV}$ & Fig cryptic virus & FR687854 & FR687855 \\
\hline 25 & FpV1 & Fusarium poae virus 1 & AF047013 & AF015924 \\
\hline 26 & FsV1 & Fusarium solani virus 1 & D55668 & D55669 \\
\hline 27 & FvBV & Flammulina velutipes browning virus & AB465308 & AB465309 \\
\hline 28 & GaRV-MS1 & Gremmeniella abietina RNA virus MS1 & AY089993 & AY089994 \\
\hline 29 & HetPV1 & Heterobasidion partitivirus 1 & HQ541323 & HQ541324 \\
\hline 30 & HetPV2 & Heterobasidion partitivirus 2 & HM565953 & HM565954 \\
\hline 31 & HetPV3 & Heterobasidion partitivirus 3 & FJ816271 & FJ816272 \\
\hline 32 & HetPV7 & Heterobasidion partitivirus 7 & JN606091 & JN606090 \\
\hline 33 & HetPV8 & Heterobasidion partitivirus 8 & JX625227 & JX625228 \\
\hline 34 & HTCV2 & Hop trefoil cryptic virus 2 & JX971980 & JX971981 \\
\hline 35 & OPV1 & Ophiostoma partitivirus 1 & AM087202 & AM087203 \\
\hline 36 & PepCV1 & Pepper cryptic virus 1 & JN117276 & JN117277 \\
\hline 37 & PepCV2 & Pepper cryptic virus 2 & JN117278 & JN117279 \\
\hline 38 & PerCV & Persimmon cryptic virus & HE805113 & HE805114 \\
\hline 39 & PmV1 & Primula malacoides virus 1 & EU195326 & EU195327 \\
\hline 40 & PoV1 & Pleurotus ostreatus virus 1 & AY533038 & AY533036 \\
\hline 41 & PsV-F & Penicillium stoloniferum virus $F$ & AY738336 & AY738337 \\
\hline 42 & PsV-S & Penicillium stoloniferum virus $S$ & AY 156521 & AY156522 \\
\hline 43 & RCCV1 & Red clover cryptic virus 1 & KF484724 & KF484725 \\
\hline 44 & RCCV2 & Red clover cryptic virus 2 & JX971978 & JX971979 \\
\hline 45 & RHsdRV2 & Rhizoctonia solani dsRNA virus 2 & KF372436 & KF372437 \\
\hline 46 & RHsV717 & Rhizoctonia solani virus 717 & AF 133290 & AF133291 \\
\hline 47 & RnPV1 & Rosellinia necatrix partitivirus 1 & AB113347 & AB113348 \\
\hline 48 & RnPV2 & Rosellinia necatrix partitivirus 2 & AB569997 & AB569998 \\
\hline 49 & RoCV1 & Rose cryptic virus 1 & EU413666 & EU413667 \\
\hline 50 & $\mathrm{RsCV} 1$ & Raphanus sativus cryptic virus 1 & AY949985.2 & DQ181926 \\
\hline 51 & $\mathrm{RsCV} 2$ & Raphanus sativus cryptic virus 2 & DQ218036 & DQ218037 \\
\hline 52 & $\mathrm{RsCV} 3$ & Raphanus sativus cryptic virus 3 & FJ461349 & FJ461350 \\
\hline 53 & SsPV1 & Sclerotinia sclerotiorum partitivirus 1 & JX297511 & JX297510 \\
\hline 54 & SsPV-S & Sclerotinia sclerotiorum partitivirus $S$ & GQ280377 & GQ280378 \\
\hline 55 & UvPV1 & Ustilaginoidea virens partitivirus 1 & KC503898 & KC503899 \\
\hline 56 & UvPV2 & Ustilaginoidea virens partitivirus 2 & KF361014 & KF361015 \\
\hline 57 & VCV & Vicia cryptic virus & AY751737 & AY751738 \\
\hline 58 & VdPV1 & Verticillium dahliae partitivirus 1 & KC422244 & KC422243 \\
\hline 59 & WCCV1 & White clover cryptic virus 1 & AY705784 & AY705785 \\
\hline 60 & WCCV2 & White clover cryptic virus 2 & JX971976 & JX971977 \\
\hline 61 & & Human Picobirnavirus & AB186898 & - \\
\hline 62 & & Otarine picobirnavirus & JQ776552 & - \\
\hline
\end{tabular}

*Informations on No. 4 - No. 60 are obtained from Nibert et al ., 2014. 